\title{
A framework for halal knowledge metadata representations
}

\begin{abstract}
End users and consumers of halal industry are facing difficulties in finding verified halal information. This occurred due to information that is stored in silos at every point of activity for every process chain, employing different structures and models, creating an issue of information verification. Integration of multiple information systems generally aims at combining selected systems so that information can be easily retrieved and manage by users. A proposed five components metadata representation development methodology is presented in this paper so that they form a unified new whole and give users the illusion of interacting with one single information system, therefore, data can be represented using the same abstraction principles (unified global data model and unified semantics) without any physical restructuring.
\end{abstract}

Keyword: Data integration; Knowledge representations; Metadata modelling; Ontology 